\title{
Purification, Characterization and Sequence Analyses of the Extracellular Giant Hemoglobin from Oligobrachia mashikoi
}

\author{
Taro Nakagawa ${ }^{1}$, Seiko Onoda ${ }^{1}$, Masaaki Kanemori ${ }^{1}$, Yuichi Sasayama ${ }^{2}$ \\ and Yoshihiro Fukumori ${ }^{1 *}$ \\ ${ }^{1}$ Department of Life Science, Graduate School of Natural Science and Technology, \\ Kanazawa University, Kakuma-machi, Kanazawa 920-1192, Japan \\ ${ }^{2}$ Noto Marine Laboratory, Institute of Nature and Environment Technology, \\ Kanazawa University, Kakuma-machi, Kanazawa 920-1192, Japan
}

\begin{abstract}
We purified an extracellular hemoglobin with the molecular mass of ca. $440 \mathrm{kDa}$ from the whole homogenates of Oligobrachia mashikoi (phylum Pogonophora) by a one-step gel-filtration. The preparation was pure to be crystallized. The $P_{50}$ values of the hemoglobin and the fresh blood prepared from O. mashikoi were about 0.82 Torr and 0.9 Torr, respectively, which were much lower than the $P_{50}$ value of human hemoglobin. However, the $n$ values of the hemoglobin and the blood were about 1.2 and 1.1, respectively.Using the improved tricine SDS-PAGE, we could separate 0 . mashikoi hemoglobin into four kinds of the globin chains, $A 1, A 2, B 1$ and $B 2$, and succeeded for the first time in cloning and sequencing of the complete cDNA encoding B1 globin gene, in addition to A1, A2 and B2 globin genes in full length. We found that all globin genes have the extracellular signal sequences in each molecule and the distal His of the B1 globin chain is replaced to Gln. Finally, we constructed phylogenetic trees of the hemoglobins from Pogonophora, Vestimentifera and Annelida.
\end{abstract}

Key words: Oligobrachia mashikoi, extracellular giant hemoglobin, purification, crystallization, oxygen binding property

\section{INTRODUCTION}

Oligobrachia mashikoi (phylum Pogonophora) lives in black mud smelling hydrogen sulfide on the shallow sea bottom at a $25 \mathrm{~m}$ in Tsukumo Bay of Noto Peninsula, Ishikawa Prefecture, Japan (Imajima, 1973). They lack a mouth, gut and anus (Sasayama et al., 2003) and Pogonophora as $O$. mashikoi possess easy closed blood-vascular system containing the extracellular giant hemoglobin (Ivanov, 1963). In general, the marine invertebrates as Pogonophora, Vestimentifera and Annelida have the extracellular giant hemoglobins (=erythrocruorins, widely including chlorocruorins), which are directly dissolved in blood or coelomic fluid (not in the erythrocyte) at high concentration (Weber and Vinogradov, 2001). There are two types of the giant respiratory proteins with the molecular masses of ca. $3000 \sim 3600 \mathrm{kDa}$ (derived from Annelida and Vestimentifera) and ca. 350 440 kDa (derived from Pogonophora and Vestimentifera) (Svedberg and Hedenius, 1934; Vinogradov, 1985; Terwilliger et al., 1987; Suzuki et al., 1988; Yuasa et al., 1996; Zal et al.,

\footnotetext{
${ }^{*}$ Corresponding author. Phone: +81-76-264-6231;

Fax : +81-76-264-6230;

E-mail: fukumor@kenroku.kanazawa-u.ac.jp
}

1996a; Weber and Vinogradov, 2001; Suzuki and Vinogradov, 2003). Recently, it has been reported that the ca. 3000 3600 kDa hemoglobin found in blood from Annelida and Vestimentifera consists of many heme-containing globin chains and heme-deficient linker proteins with a hexagonalbilayer quaternary structure proposed as "bracelet model" (Vinogradov, 1985; Vinogradov et al., 1986; de Hass et al., 1996; Weber and Vinogradov, 2001). Moreover, the X-ray crystal structural analyses at a $5.5 \AA$ resolution of the Annelida Lumbricus terrestris erythorocruorin (ca. 3000 3600 $\mathrm{kDa}$ ) clearly indicate that this hemoglobin is assembled from 144 globin chains (twelve dodecamers) and 36 linker chains (twelve trimer linker subunits) with a hexagonal-bilayer quaternary structure (Royer Jr et al., 2000). However, the threedimensional structure of the ca. 350 440 kDa hemoglobin found in blood from Pogonophora and Vestimentifera have not been reported until now, although this hemoglobin has been proposed to be a dimer of dodecamers (Zal et al., 1996b; Weber and Vinogradov, 2001) and possess a small ring-shaped structure based on the analyses of a transmission electron microscopy (Zal et al., 1996a).

On the other hand, it is generally accepted that Pogonophora and Vestimentifera keep endosymbionts in the poste- 
rior specific organ called as "trophosome" or "bacteriocyte" (Cavanaugh et al., 1981; Southward, 1982; Matsuno and Sasayama, 2002; Kimura et al., 2003). The extracellular hemoglobins from Pogonophora and Vestimentifera show high oxygen binding affinities (Wells and Dales, 1976; Terwilliger et al., 1987; Arp et al., 1990) and can simultaneously transport not only oxygen with heme for mitochondrial aerobic respiratory chain but also hydrogen sulfide for the symbiotic bacteria which utilize sulfides as primary electron donor in an anaerobic respiratory chain (Felbeck, 1981; Arp et al., 1983, 1987; Nelson and Fisher, 1995; Zal et al., 1997, 1998; Weber and Vinogradov, 2001). Therefore, it is quite likely that a ca. $350 \sim 440 \mathrm{kDa}$ giant hemoglobin from Pogonophora $O$. mashikoi functions as not only oxygen transporter to the hypoxia posterior end of the self-body in mud but also sulfide transporter to the endosymbiotic bacteria in the bacteriocyte.

In 1996, Yuasa et al. reported that the O. mashikoi giant hemoglobin is composed of eight kinds of globin chains (a1 a5, b, c, d) based on the analyses of electrospray ionization mass spectrometry and sequenced the three major globin genes encoding A2 (a5), A1 (b), and B2 (c), respectively (Yuasa et al., 1996). However, until now, the sequence of B1 (d) globin gene and the sequences of four globin genes from initiation codon to stop codon have not been reported. In the present study, we purified the $O$. mashikoi giant hemoglobin to be crystallized and characterized oxygen affinity. Furthermore, we succeeded in the cloning and sequencing of the four globin genes in the full length and constructed the phylogenetic trees from Pogonophora, Vestimentifera and Annelida hemoglobins including $O$. mashikoi B1 globin chain identified in the present study.

\section{MATERIALS AND METHODS}

\section{Beard worm collections}

Specimens of $O$. mashikoi were dredged from the shallow sea bottom at a depth of $25 \mathrm{~m}$ in Tsukumo Bay of the Noto peninsula in Japan $\left(37^{\circ} 18^{\prime} \mathrm{N}, 137^{\circ} 14^{\prime} \mathrm{E}\right)$. We carefully collected the beard worms living in smelly mud including hydrogen sulfide with a special dredger made $7 \mathrm{~mm}$-thick iron into a box type, $40 \times 40 \times 70 \mathrm{~cm}$, about $80 \mathrm{~kg}$ weight on land (Sasayama et al., 2003) and transferred them into the fresh seawater on the ship. After the several collections, we took their bodies out of a self-made chitinous tube into the seawater by a syringe. All their bodies were immediately frozen in liquid nitrogen and stored at $-80^{\circ} \mathrm{C}$ until use.

\section{Physical and chemical measurements}

The spectra of the hemoglobin were recorded with UV-265FS recording spectrophotometer (Shimadzu, Co., Kyoto, Japan). The protein content was determined by the methods of Lowry (Lowry et al., 1951) with bovine serum albumin as a standard. The heme content was determined on the basis of the molar coefficient of pyridine ferrohemochrome; $\varepsilon_{550 \mathrm{~nm}}=34,400 \mathrm{M}^{-1} \mathrm{~cm}^{-1}$ for the ferrohemochrome of heme $b$ (Falk, 1964). The molecular mass of $O$. mashikoi hemoglobin was estimated with a HiPrep 16/60 Sephacryl S-300 $\mathrm{HR}$ (Amersham Biosciences) equilibrated with $50 \mathrm{mM}$ Tris- $\mathrm{HCl}$ buffer, pH 7.5 containing $0.2 \mathrm{M} \mathrm{NaCl}$. The apoferritin $(443 \mathrm{kDa})$, alcohol dehydrogenase (150 kDa) and carbonic anhydrase (29
$\mathrm{kDa}$ ) were used as molecular mass calibrants.

\section{Crystallization of $\boldsymbol{O}$. mashikoi hemoglobin}

O. mashikoi hemoglobin was crystallized by hanging drop vapor diffusion method. The mixture of the hemoglobin solution and the reservoir solution were stilly incubated at $10^{\circ} \mathrm{C}$ for a month.

\section{Measurements of oxygen binding properties of the purified hemoglobin and the blood}

Oxygen equilibrium curve of $O$. mashikoi hemoglobin was obtained by the spectrophotometric method with slight modifications (Matsukawa et al., 1979). The heme concentration of the hemoglobin solution was adjusted to be $50 \mu \mathrm{M}$ heme with $0.5 \mathrm{M}$ phosphate buffer, $\mathrm{pH}$ 7.0. We obtained the deoxygenated hemoglobin by repeating evacuations and flushing with Argon gas (99.99\%) in a Thunberg-type cell with a $1 \mathrm{~cm}$ light path. It should be noted that $O$. mashikoi hemoglobin is denatured during evaculation in $0.1 \mathrm{M}$ phosphate buffer, pH 7.0. So, we prepared the hemoglobin solution with $0.5 \mathrm{M}$ phosphate buffer, $\mathrm{pH}$ 7.0. Then the small amount of air was added to the hemoglobin for oxygenation and the absorption spectra were measured from $450 \mathrm{~nm}$ to $650 \mathrm{~nm}$ at $25^{\circ} \mathrm{C}$. The $\mathrm{pH}$ of the hemoglobin solution was always measured after the end of each experiment. The $P_{50}$ value and the Hill coefficient, $n$ value, were calculated from the Hill plot. The measurement of the oxygen dissociation curve was also made with the whole blood, which was freshly prepared from the unfrozen body and diluted to be $50 \mu \mathrm{M}$ heme with $0.5 \mathrm{M}$ phosphate buffer, $\mathrm{pH}$ 7.0. Met-hemoglobin was not produced during oxygen equilibrium experiments because an absorption peak at $630 \mathrm{~nm}$ was not detected.

\section{Electrophoresis analyses}

Improved tricine SDS-polyacrylamide gel electrophoresis (PAGE) system for good separation of the proteins with low molecular mass was adapted to the methods of the references (Schägger and von Jagow, 1987; Fountoulakis et al., 1998). The disk tricine SDS-PAGE $(18.2 \% \mathrm{~T}, 3 \% \mathrm{C}$ containing $5.8 \mathrm{M}$ urea) was performed with the separating gel of $18 \mathrm{~cm}$ high, $7 \mathrm{~mm}$ diameter and the stacking gel of $1 \mathrm{~cm}$ high. The hemoglobin sample was boiled with $2 \%$ $(\mathrm{w} / \mathrm{v})$ SDS plus $0.05 \%(\mathrm{v} / \mathrm{v}) \beta$-mercaptoethanol for $5 \mathrm{~min}$. The disk tricine SDS-PAGE was performed at $5 \mathrm{~mA}$ constant current per one disk at room temperature. The protein bands were fixed and stained with $18.3 \%(\mathrm{v} / \mathrm{v})$ methanol solution containing $6.4 \%(\mathrm{v} / \mathrm{v})$ acetic acid, $6 \%(\mathrm{w} / \mathrm{v})$ trichloroacetic acid and $0.02 \%(\mathrm{w} / \mathrm{v})$ Coomassie brilliant blue R-250 for overnight by shaking. The stained gels were completely destained with $28.6 \%(\mathrm{v} / \mathrm{v})$ methanol solution containing $4.8 \%(\mathrm{v} / \mathrm{v})$ acetic acid for overnight by shaking.

The slab tricine SDS- PAGE (20\%T and 3\%C containing 4.89 $M$ urea) was performed with the separating gel of $110 \mathrm{~mm}$ high, 135 $\mathrm{mm}$ wide and $1.0 \mathrm{~mm}$ thick, and the stacking gel of $10 \mathrm{~mm}$ high. After the hemoglobin solution was boiled with $2 \%(w / v)$ SDS plus $0.05 \%(\mathrm{v} / \mathrm{v}) \beta$-mercaptoethanol for $5 \mathrm{~min}$, solid urea was added to the sample to be $1 \mathrm{M}$ as final concentration. The slab tricine SDSPAGE was performed at $20 \mathrm{~mA}$ constant current in the stacking gel and at $30 \mathrm{~mA}$ constant current in the separating gel at room temperature. The protein bands were fixed and stained with $45 \%(\mathrm{v} / \mathrm{v})$ methanol solution containing $10 \%(\mathrm{v} / \mathrm{v})$ acetic acid and $0.25 \%(\mathrm{w} / \mathrm{v})$ Coomassie brilliant blue G-250 for $30 \mathrm{~min}$ by shaking. The stained gels were completely destained with $26 \%(\mathrm{v} / \mathrm{v})$ methanol solution containing $8.2 \%(\mathrm{v} / \mathrm{v})$ acetic acid for $3 \mathrm{~h}$ by shaking and the protein bands were transferred to polyvinylidene fluoride membranes (Sequi-Blot ${ }^{T M}$ PVDF membrane, Bio-Rad, USA). The N-terminal amino acid sequences of all globin chains were determined with the protein sequencer model 476A (Applied Biosystems, USA).

\section{Cloning and sequencing techniques}

All general DNA manipulations were performed according to Sambrook et al. (Sambrook et al., 1989). Total RNA was purified 
from O. mashikoi with ISOGEN (NIPPON GENE, Japan) by the method as described in the instructions attached. The degenerate oligonucleotide of $\mathrm{B} 1$ globin chain was designed based on the $\mathrm{N}$ terminal amino acid sequences (E-V-V-I-S-E-W and E-W-D-Q-V-FN) as follows; Degenerate-B1-f1: 5'-GARGTNGTNATHAGYGARTGG-3', Degenerate-B1-f2: 5'-GARGTNGTNATHTCNGARTGG3', and Degenerate-B1-f3: 5'-GARTGGGAYCARGTNTTYAAY-3', (where $R$ represents $A$ and $G ; Y$ represents $C$ and $T ; H$ represents $\mathrm{A}, \mathrm{C}$ and $\mathrm{T} ; \mathrm{N}$ represents $\mathrm{A}, \mathrm{C}, \mathrm{G}$ and T.) Reverse transcriptasePCR (RT-PCR) was performed with these three primers and oligo$\mathrm{dT}_{20}$ primer by using Revertra Ace reverse transcriptase (TOYOBO, Japan) and KOD-plus DNA polymerase (TOYOBO, Japan). The PCR-products were inserted to pUC119 vector digested with Smal. These recombinant plasmids were transformed into Escherichia coli XL-1 Blue MRF' (STRATAGENE, USA) and identified with ABI PRISM BigDye Terminator v3.0 Ready Reaction Cycle Sequencing Kit (Applied Biosystems, USA) by using a multi capillary DNA sequencer, ABI PRISM 3100 DNA Analyzer (Applied Biosystems, USA).

The rapid amplification of 5'CDNA ends (5'-RACE) of A1, A2, B1, and B2 globin genes was performed with 5'-Full RACE Core Set (TAKARA, Japan). For the first-strand cDNA synthesis using AMV Reverse Transcriptase XL (Life Sciences, USA) and Revertra Ace reverse transcriptase (TOYOBO, Japan), the oligonucleotides of the globin chains were designed with 5'-phosphate as follows; 5'pRACE-A1-r: 5'p-TTAGCCGGAAATACCGCTAGC-3', 5'pRACEA2-r: 5'p-TTAACCAGAAATGCCGCTGAC-3', 5'pRACE-B1-r: 5'pCATCGATGTGAGCGCTAGGAA-3', and 5'pRACE-B2-r: 5'p-TTACAAGCCTGCTGAGATGC-3'. The first-strand cDNAs were ligated with T4 RNA ligase after dealing with RNase $\mathrm{H}$, and formed the concatemer or circular DNAs. The oligonucleotides for the inversePCR were designed as follows; InversePCR-A1-f: 5'-TGATCTTGCTCACCTTGCTGG-3', InversePCR-A2-f: 5'-TGAATTCTCAGCATGACTCTCG-3', InversePCR-B1-f: 5'-CCTGCTGTCACATTTGAACGC-3', InversePCR-B2-f: 5'-TGCTCATTTCGATGTGATGGC-3', InversePCR-A1-r: 5'-GAGCTGACTGATCAGACGGTTAAG-3', InversePCR-A2-r: 5'-GCGTTGAGGACAGGTACGTC-3', InversePCRB1-r: 5'-CCAGAAACCACACGGGATAC-3', and InversePCR-B2-r: 5'-TCGTTGAGCATGTTAATGGCG-3'. The oligonucleotides for the nested-PCR were designed as follows; NestedPCR-A1-f: 5'-TTTGGATGCATCCAACTTCG-3', NestedPCR-A2-f: 5'-ATATCCAGCATTCGTCGCTTC-3', NestedPCR-B1-f: 5'- CTGTTACCCATTTAAGTCAGGC-3', NestedPCR-B2-f: 5'-TGTGATGGCTGAGGCTTTCG-3', NestedPCR-A1-r: 5'-TAAGAGCTCCAGTGACACGAAGG-3', NestedPCR-A2-r: 5'-CACACATGTCAAGACCACCC-3', NestedPCR-B1r: 5'-GTTGTTACTGTCACCACCACCG-3', and NestedPCR-B2-r: 5'TCGAGACCGTTAACAACGCG-3'.

Inverse-PCR and nested-PCR were performed with KOD-plus DNA polymerase (TOYOBO, Japan). The subcloning and DNA sequencing were carried out by the same method as described above.

\section{Informative search, multiple alignment and phylogenetic analy- ses}

The amino acid sequences and DNA sequences of the globin chains from O. mashikoi were analyzed with BLAST program (http:/ /www.ncbi.nih.gov/BLAST/) for homology search. The amino acid sequences of the extracellular giant hemoglobins from Pogonophora, Vestimentifera, Annelida and Mollusca, human hemoglobin and myoglobin were obtained from the Entrez Protein program (http://www.ncbi.nlm.nih.gov/entrez/index.html) and from the reference (Suzuki et al., 1995). The structures of hemoglobins were observed with MMDB and Entrez Structure program (http:// www.ncbi.nih.gov/Structure/). Signal sequences were predicted with PSORT II program in ExPASy (http://psort.nibb.ac.jp/ from2.html). The multiple alignment of the hemoglobins from Pogonophora, Vestimentifera, Annelida and Mollusca, human hemoglobin and myoglobin was constructed with Clustal $X 1.83$ program (http://www-igbmc.u-strasbg.fr/Biolnfo/ClustalX/Top.html). PHYML 2.4.1 program (http://atgc.lirmm.fr/phyml/) was used for construction of the maximum likelihood (ML) tree with an input generated tree of BIONJ and with the calculated pairwise ML distance using WAG amino acid substitution model (Whelan and Goldman, 2001). PHYML 2.4.1 program also computed a discrete gamma distribution of eight variable rate categories for rate-across-site variation containing the estimated optimum shape parameter (alpha) and invariable sites, and with the bootstrap resampling at 100 times, respectively. The tree was drawn by $\mathrm{NJ}$-plot program (http:// pbil.univ-lyon1.fr/software/njplot.html).

A

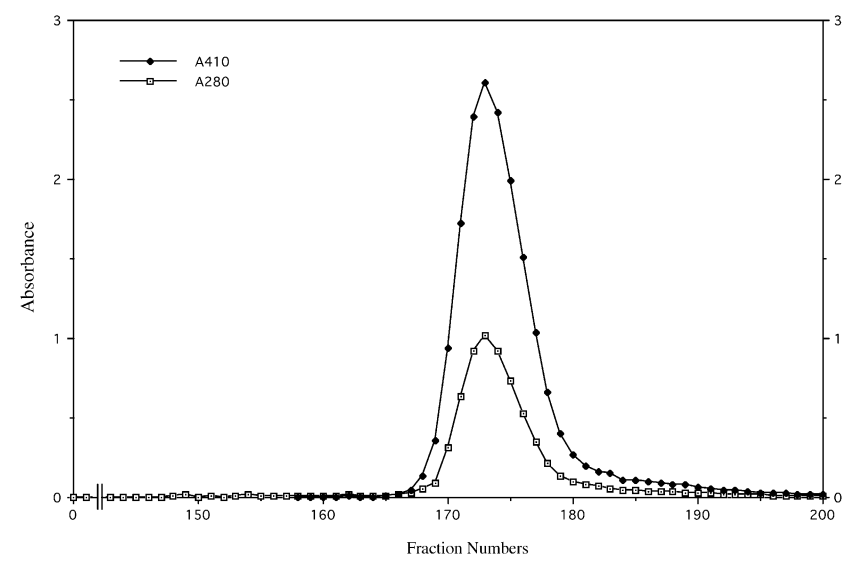

B

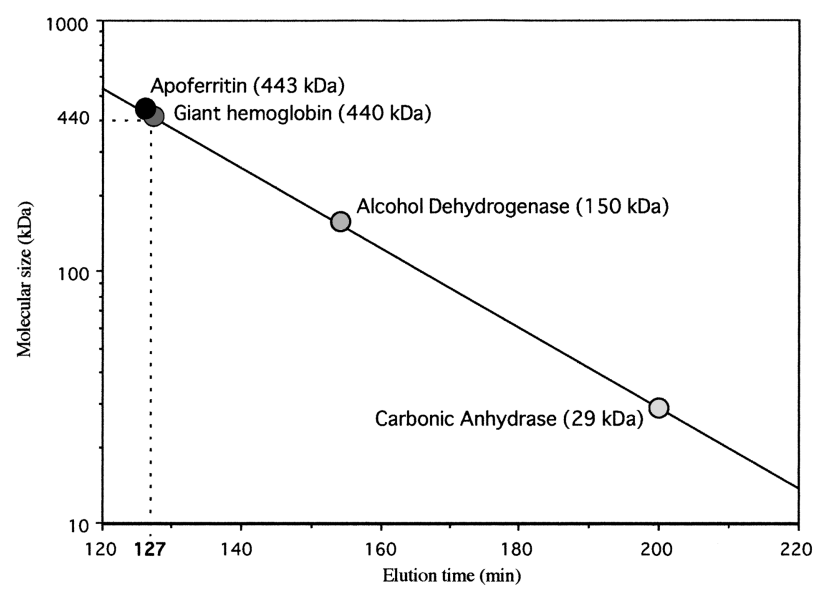

Fig. 1. Elution profile and molecular mass estimation of $O$. mash$i k o i$ hemoglobin. (A) Elution profile of the O. mashikoi hemoglobin by gel filtration with a Sephacryl S-300 column. The column $(95 \times 2.2$ $\mathrm{cm})$ was equilibrated and eluted with $50 \mathrm{mM}$ Tris- $\mathrm{HCl}$ buffer $(\mathrm{pH} 7.5)$ containing $0.2 \mathrm{M} \mathrm{NaCl}$. The flow rate was $1.5 \mathrm{ml} / 15 \mathrm{~min}$ in each tube. The hemoglobin fractions with a constant ratio of A415 / A280 $=2.5 \sim 3.0$ were collected (fraction numbers; 170 178) and used for the experiment of crystallization, oxygen binding assay, and SDSPAGE. (B) Molecular mass estimation of the $O$. mashikoi giant hemoglobin, using gel filtration with a Sephacryl S-300 column. The elution buffer was $50 \mathrm{mM}$ Tris- $\mathrm{HCl}$ buffer $(\mathrm{pH} 7.5)$ containing $0.2 \mathrm{M}$ $\mathrm{NaCl}$. The proteins used as the markers were: apoferritin $(443 \mathrm{kDa})$, alcohol dehydrogenase (150 kDa) and carbonic anhydrase (29kDa). 


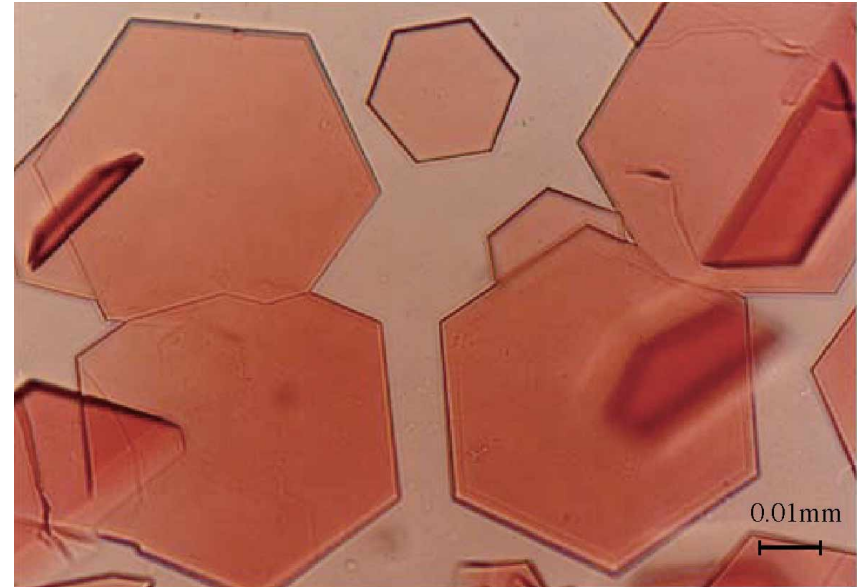

Fig. 2. The photomicrograph of $O$. mashikoi hemoglobin crystals. The crystallization of $O$. mashikoi hemoglobin was performed with hanging drop vapor diffusion method. The well-shaped hexagonal crystals with the size of about $50 \mu \mathrm{m}$ width were prepared from the O. mashikoi hemoglobin solution.

\section{A}

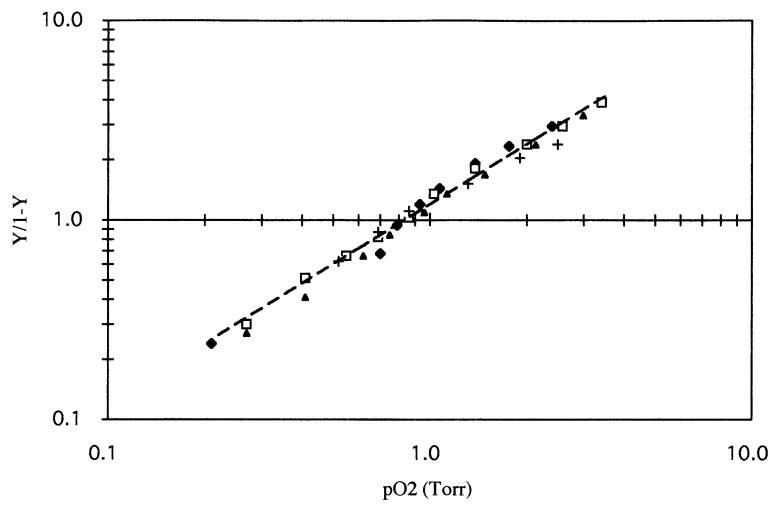

B

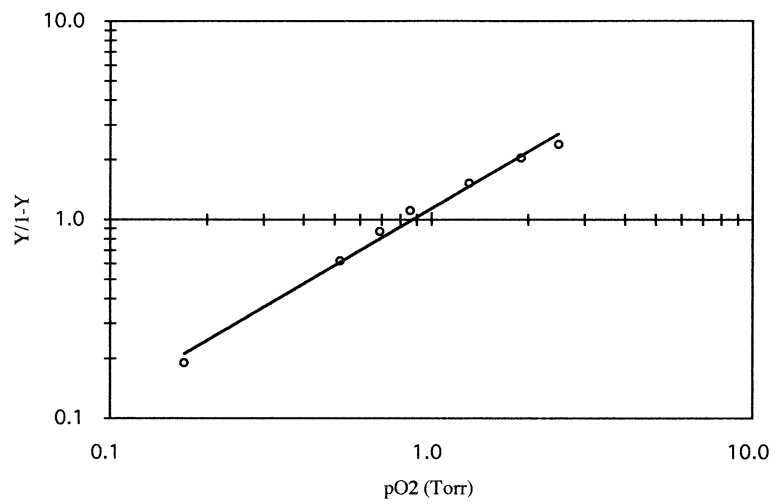

Fig. 3. The oxygen dissociation curves of the purified hemoglobins and the blood of O. mashikoi. (A) Hill plot of the O. mashikoi hemoglobins. The hemoglobins purified were diluted with $0.5 \mathrm{M}$ phosphate buffer, $\mathrm{pH} 7.0$ to $50 \mu \mathrm{M}$ heme. Four oxygen dissociation experiments were independently performed by the method as described in Materials and Methods $(\square, \boldsymbol{\Delta}, \boldsymbol{\Delta},+)$. (B) Hill plot of the blood from $O$. mashikoi. The blood was freshly prepared from the about fifty individuals of $O$. mashikoi and diluted with $0.5 \mathrm{M}$ phosphate buffer, $\mathrm{pH} 7.0$ to $50 \mu \mathrm{M}$ heme. The titration was performed by the method as described in MATERIALS AND METHODS.

\section{RESULTS}

\section{Purification and crystallization of the giant hemoglobin from O. mashikoi}

All purification steps were conducted at $4^{\circ} \mathrm{C}$. After the $O$. mashikoi bodies were homogenized with a tefron homogenizer on ice, the homogenate was centrifuged at 22,000 $\times g$ for $1 \mathrm{~h}$. The supernatant obtained was concentrated by ultrafiltration with 100,000 MW cut membrane (VIVASPIN $20 \mathrm{ml}$ CONCENTRATOR, 100,000 MW cut, VIVASCIENCE, U.K.). The concentrated blood was subjected to gel filtration with a Sephacryl S-300 HR (Amersham Biosciences) column $(95 \times 2.2 \mathrm{~cm})$ equilibrated with $50 \mathrm{mM}$ Tris- $\mathrm{HCl}$ buffer, $\mathrm{pH} 7.5$ containing $0.2 \mathrm{M} \mathrm{NaCl}$. Fig. 1 (A) shows the elution profile by a Sephacryl S-300 column chromatography. We observed one peak with high absorbances at 280 and 410 $\mathrm{nm}$ on the column chromatography. Following each of the absorbances at $280 \mathrm{~nm}$ and $410 \mathrm{~nm}$, the fractions with a constant ratio of the absorbance at $410 \mathrm{~nm} / 280 \mathrm{~nm}$ of 2.5 to 3.0 were collected and concentrated to be ca. $50 \mathrm{mg}$ protein/ml by ultrafiltration with $10,000 \mathrm{MW}$ cut membrane (MICROCON YM-10, 10,000 MW cut, MILLIPORE, USA) and stored at $-80^{\circ} \mathrm{C}$ until use. The molecular size of $O$. mashikoi hemoglobin was estimated to be about $440 \mathrm{kDa}$ by a Sephacryl S-300 column chromatography (Fig. 1 (B)).

To confirm the purity of the $O$. mashikoi hemoglobin, we tried crystallization by the hanging drop vapor diffusion method. Fig. 2 shows a photomicrograph of the crystals of the $O$. mashikoi hemoglobin. The crystals were obtained

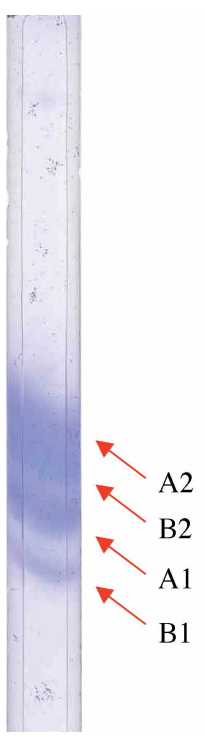

\author{
A2: D-X-T-S-L-N-R-L-L-V-K-R-Q-W-A-E-A-Y-G-E \\ B2: S-S-X-X-S-S-E-D-R-X-N-V-M-H-N-X-W-D-A-A \\ A1: V-X-N-R-L-E-Q-I-L-V \\ B1: E-X-N-S-R-G-D-A-E-V-K-I-S-E-W-D-Q-V-F-N \\ $\mathrm{X}$ : unidentified amino acid residue
}

Fig. 4. Improved disk tricine SDS-PAGE of the O. mashikoi hemoglobin. The SDS-PAGE $(18.2 \% \mathrm{~T}, 3 \% \mathrm{C}$ containing $5.8 \mathrm{M}$ urea) was performed by the method as described in MATERIALS AND METHODS. The hemoglobin sample was boiled with $2 \%(w / v)$ SDS plus $0.05 \%(\mathrm{v} / \mathrm{v}) \beta$-mercaptoethanol for $5 \mathrm{~min}$. The protein bands were calibrated with Precision Plus Protein Standards marker (Bio-Rad, USA). The N-terminal amino acid sequences of four globin chains determined by the protein sequencer are shown in the figure. $X$ : unidentified amino acid residue. 
under the experimental condition as follows; the reservoir solution containing $0.1 \mathrm{M}$ imidazole, $\mathrm{pH} 6.5$ and $1.0 \mathrm{M}$ sodium acetate trihydrate and the hemoglobin solution (85 $\mathrm{mg} / \mathrm{ml}$ ) in $50 \mathrm{mM}$ Tris- $\mathrm{HCl}$ buffer, $\mathrm{pH} 8.0$ containing $0.2 \mathrm{M}$ $\mathrm{NaCl}$ were suspended at the ratio $1: 1$ and stilly incubated at $10^{\circ} \mathrm{C}$ for a month.

\section{Oxygen binding properties of the purified hemoglobin and the blood freshly prepared from 0 . mashikoi}

The respiratory properties of $O$. mashikoi hemoglobin and the blood were analyzed by the method as described in Materials and Methods. Fig. 3 (A) shows the Hill plot of the purified $O$. mashikoi hemoglobin. The $P_{50}$ value and $n$ value were estimated to be about 0.82 Torr and 1.2. Fig. 3 (B) shows the Hill plot of the fresh blood prepared from $O$. mashikoi. The $\mathrm{P}_{50}$ value and $n$ value were estimated to be about 0.9 Torr and 1.1, respectively.

\section{Subunit structure and the complete amino acid sequences of four globin chains from $O$. mashikoi hemo- globin}

The subunit structure of the $O$. mashikoi hemoglobin was analyzed by disk tricine SDS-PAGE. Fig. 4 shows the results of disk tricine SDS-PAGE. These apparent molecular sizes were estimated to be ca. 14-16 kDa. After the slab tricine SDS-PAGE, the four protein bands were transferred from the gel to the PVDF membrane and then, their partial
$\mathrm{N}$-terminal amino acid sequences were determined by the protein sequencer. As shown in Fig. 4, the N-terminal amino acid sequences of four globin chains were consistent with the registered sequences of the $A 1, A 2, B 1$, and $B 2$ globin chains in the database.

In the present study, we succeeded in sequencing four globin cDNAs in full length by RT-PCR and 5' RACE methods and deduced the full amino acid sequences (Accession numbers; A1; AB185392, A2; AB185391, B1; AB185394 and $B 2 ; A B 185393$. Fig. 5 shows the alignment of the deduced complete amino acid sequences of four globin chains. Gaps have been inserted where necessary to provide maximum alignment of these sequences. The $\mathrm{N}$-terminal amino acid sequence of the A2 (a5) globin chain was determined to be D-X-T-S-L-N-R-L-L-V-K-R-Q-W-A-E-A-Y$\mathrm{G}-\mathrm{E}$ by protein sequencer, suggesting that the 16 amino acid residues from first Met to Ala (M-K-S-L-I-V-F-A-C-L-VA-Y-A-A-A) may be a signal peptide. Therefore, the A2 (a5) globin chain consists of 142 amino acid residues with the molecular mass of 15,312 Da. Similarly, the A1 (b) globin chain has a signal peptide (M-K-V-L-I-I-F-A-C-L-V-V-M-A-SA) and consists of 140 amino acid residues with the molecular mass of 15,174 Da. The B2 (c) globin chain has a signal peptide (M-I-A-L-F-V-L-M-G-L-M-A-A-A-S-A) and consists of 147 amino acid residues with the molecular mass of 15,606 $\mathrm{Da}$. The B1 (d) globin chain has a signal peptide (M-T-I-LV-L-F-L-S-C-A-A-L-A-S-A) and consists of 145 amino acid

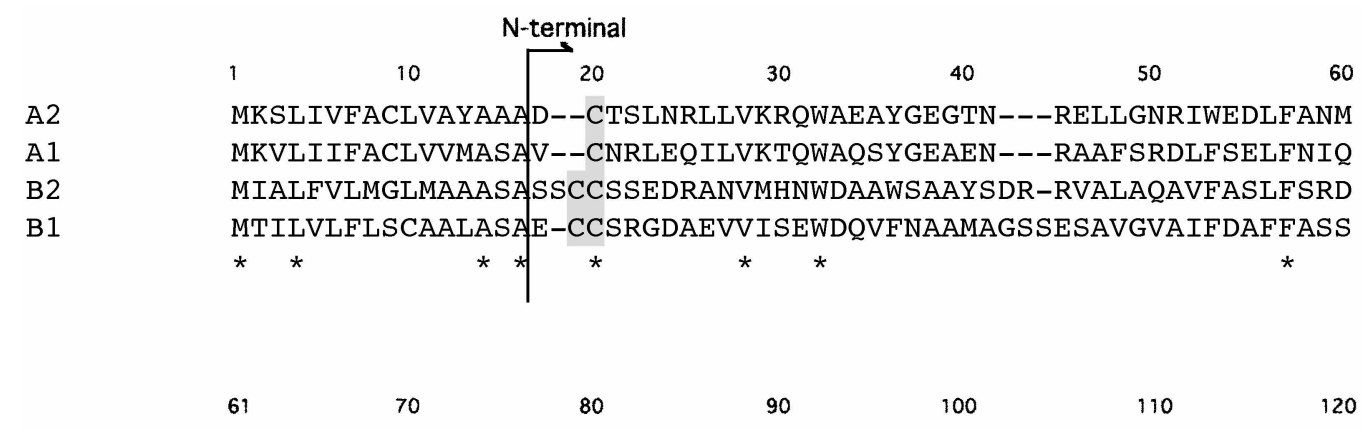

A2 PDARGLFSRVNGNDIDSSEFQAHSLRVLGGLDMCVASLDDVPVLNALLARLNSQHDSRGI A1 GSSRALFSGVGVDDMNSAAFTAH CLRVTGALNRLISQLDQQATINADLAHLAGQHASRNL B2 AAAQGLFSGVSADNPDSADFRAH CVRVVNGLDVAINMLNDPAVLNEQLAHLSAQHQARAG GVSPSMF--PGGGDSNPEFLAQVSRVVSGADIAINSLTNRATCDSLLSHLNAQHRAISG
$*$ * * * * * * * * * $121 \quad 130 \quad 140 \quad 150 \quad 160 \quad 165$

A2 PAAGYPAFVASAISAVRATVGARSFDNDAWNSCMNQIVSGISG-A1 DASNFAAMGQAVMSVVPTHL--DCFNQHAWGECYERIASGISG-B2 VAAAHFDVMAEAFAEVMPQVS-SCFSSDSWNRCFARIANGISAGL B1 VTGAAVTHLSQAISSVVAQVL-PSAHIDAWEYCMAYIAAGIGAGL

Fig. 5. Multiple alignment of amino acid sequences of four globin chains from $O$. mashikoi hemoglobin. The amino acid sequences were deduced from the cDNAs encoding the A1, A2, B1 and B2 globin genes. The amino acid residues of the four globin chains from 1st to 16th are predicted to be the extracellular secretory signal peptides. The conserved amino acid residues of the four globin chains are indicated by asterisks (*). The specific cysteine residues are shaded in gray, and the proximal and distal histidine residues are bold. These four globin chains were registered in DDBJ data bank with the accession numbers of A1:AB185392, A2:AB185391, B1:AB185394, and B2:AB185393. 
residues with the molecular mass of $14,778 \mathrm{Da}$.

\section{DISCUSSION}

\section{Purification and characterization of the 0 . mashikoi giant hemoglobin}

In the present study, we could purify the $O$. mashikoi hemoglobin from the whole homogenates in a few days, using a mild column chromatography as gel filtration. As shown in Fig. 1 (A), a symmetric peak was observed in the elution profile. Furthermore, any small proteins and free hemes were not observed in the elution profile. It should be noted that Vestimentifera Riftia pachyptila hemoglobins were easily dissociated into the small proteins when the blood was stored at $-40^{\circ} \mathrm{C}$ (Zal et al., 1996a). On the other hand, Zal et al. reported that Vestimentifera Riftia pachyptila has two kinds of the giant hemoglobin with the molecular mass of $440 \mathrm{kDa}$, which are assembled with different globin chains and localized in its blood (V2) and coelomic fluid (C1), respectively (Zal et al., 1996a, b). However, the $O$. mashikoi hemoglobin solution was highly pure enough to be crystallized as shown in Fig. 2. Therefore, it is concluded that pogonophoran O. mashikoi has one kind of $440 \mathrm{kDa}$ hemoglobin different from Vestimentifera R. pachyptila.

The subunit structure of the $O$. mashikoi hemoglobin was analyzed by the improved SDS-PAGE system. We identified the four kinds of the globin chains, A1 (b), A2 (a5), B1 (d), and B2 (c). Yuasa et al. reported that the O. mashikoi giant hemoglobin is composed of eight kinds of globin chains (a1 a5, b, c, d) based on electrospray ionization mass spectrometer (Yuasa et al., 1996). However, the intensities of a1 a4 globin chains in the MaxEnt processed spectra are lower than that of a5 (A2), and all of the molecular masses of a1 a4 globin chains are lower than that of a5 (A2). Moreover, these spectra of a1 a4 globin chains were disappeared in the reduced and carbamidomethylated condition. Therefore, the minor a1 a4 globin chains might be the degradated products from a5 (A2).

As shown in Fig. $3(A)$ and $3(B)$, the $P_{50}$ values of the purified hemoglobin and the blood are about 0.82 Torr and 0.9 at $\mathrm{pH} 7.0$, respectively. These values are much lower than that of human hemoglobin A (Nagai et al., 1972). In general, pogonophores transport the oxygen to the hypoxia posterior end of the self-body in the reductive and anaerobic black mud (Wells and Dales, 1976; Terwilliger et al., 1987). Therefore, the high affinity for oxygen of the hemoglobin seems to be physiologically important for growth. On the other hand, the Hill coefficient, $n$ value, of the purified hemoglobin is estimated to be about 1.2, suggesting that the $O$. mashikoi hemoglobin has little co-operative oxygen binding property. However, it should be noted that the O. mashikoi hemoglobin is not denatured during the purification because the fresh blood has the same the $P_{50}$ and $n$ values.

\section{Amino acid sequences of four globin chains from 0 . mashikoi hemoglobin}

Fig. 5 shows that multiple alignment of amino acid sequences of four globin chains from $O$. mashikoi. We analyzed the amino acid sequences of four globin chains of $O$. mashikoi hemoglobin, using PSORT II program, and found that they have secretory signal sequences in the $\mathrm{N}$-terminal region. The 16 amino acid residues of four globin chains were mainly occupied with the hydrophobic amino acid residues and especially, the signal sequences of A1 and A2 are well-defined consensus sequence; the hydrophobic core is preceded by basic residues as $\mathrm{K}$ and followed by a cleavage site for signal peptidase. These results are consistent with the localization of $O$. mashikoi hemoglobin.

The identity and homology of these four globin chains were calculated to be $13.5 \%$ and $46 \%$, respectively. These are much lower than those of human hemoglobins $\alpha$ and $\beta$, $42 \%$ and $78.4 \%$. However, the amino acid residues participating in the globin folding were highly conserved in the four globins. The proximal His at position 115, which occupies one of the axial positions of the heme and is in direct contact with $\mathrm{Fe}$ of the heme, was completely conserved in all the globin chains. The distal His at position 83 , which is sandwiched between the ring nitrogen of the His and the iron atom of heme, was also conserved in A1, A2 and B2 globin chains, excepting B1 globin chain replaced by Gln. The human $\alpha$ globin chain variant, $\mathrm{Hb}$ Boghe, replaced to $\mathrm{Gln}$ at the distal His site, shows the normal potential of the $\mathrm{O}_{2}$ and $\mathrm{CO}$ affinity and the normal oxygen equilibrium curves (Lacan et al., 1999). Furthermore, the distal His residues of the globin chains from Calyptogena species hemoglobin and $R$. pachyptila B1c globin chain are also replaced by Gln (Kawano et al., 2003; Bailly et al., 2002). Therefore, it is quite likely that the B1 globin chain bind oxygen as same as the A1, A2 and B2 globin chains.

Fig. 6 shows the multiple alignments of amino acid sequences of extracellular giant hemoglobins from Pogonophora, Vestimentifera including the partial amino acid sequences of $R$. pachyptila $\mathrm{A} 1, \mathrm{~B} 1 \mathrm{a} \sim \mathrm{B} 1 \mathrm{c}$ and $\mathrm{B} 2$ globin chains and Annelida, and those of intracellular hemoglobins from Annelida, Mollusca and vertebrata. The globin chains of the extracellular giant hemoglobins, interestingly, have a few Cys residues in the molecule. Recently, Strand et al. has been reported the crystal structure of the hemoglobin dodecamer from Lumbricus erythrocruorin (Strand et al., 2004). The structure reveals inter-disulfide bridges between $A 1$ and $B 2$ and between $B 1$ and B2, respectively. Therefore, the dodecamer has $A 1, B 1$ and $B 2$ trimeric structure, while A2 is not disulfide linked to other chains. Furthermore, they have found the intra-disulfide bridges in all globin chains. The Cys residues participated in such intra- and inter-disulfide bridges are also conserved in the globin chains of $O$. mashikoi hemoglobin. Therefore, the $O$. mashikoi hemoglobin may have the similar structure as that of the hemoglobin dodecamer from Lumbricus erythrocruorin.

On the other hand, $R$. pachyptila hemoglobins have 


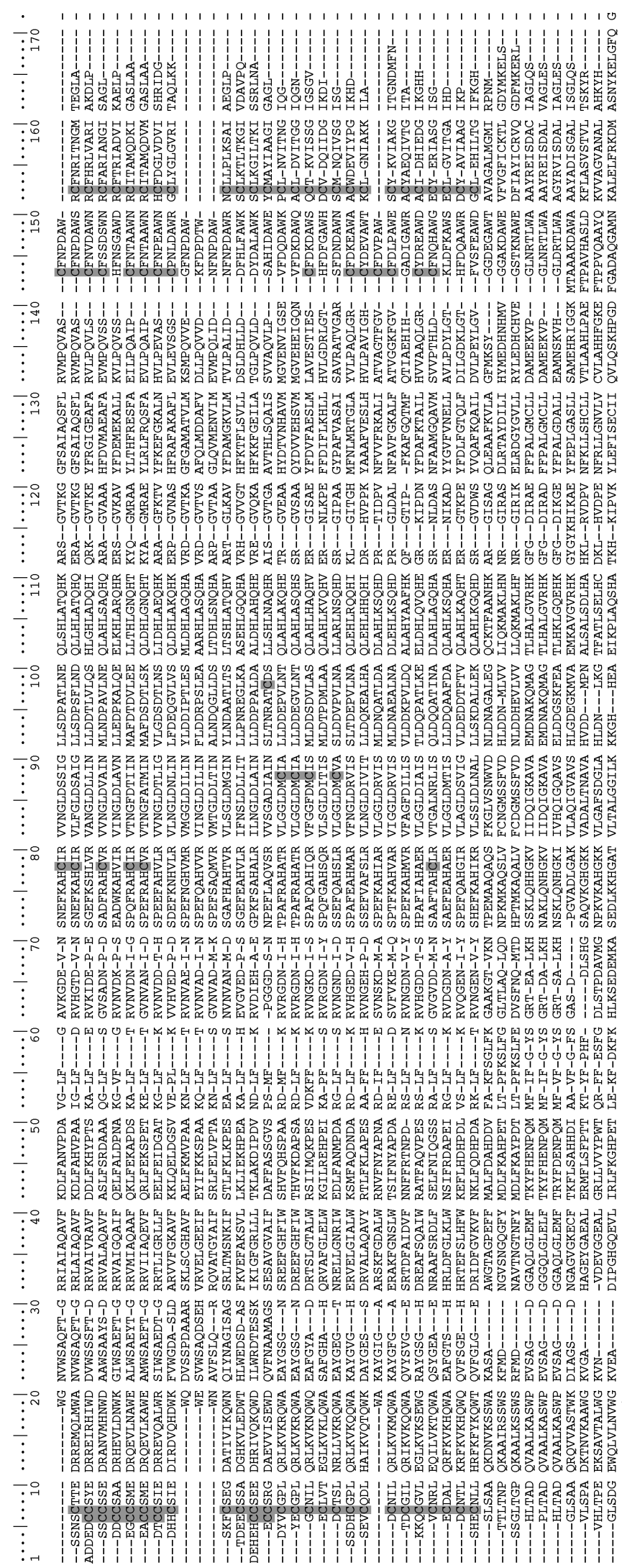

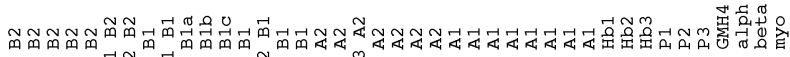

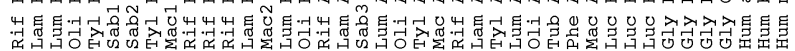

ability to bind sulphide and it is proposed that free Cys residues of the $\mathrm{Hb}$ are concerned with the sulphide binding (Zal et al., 1998). Figure 6 shows the ${ }^{78} \mathrm{Cys}$ residues of B2 globin chains and ${ }^{88}$ Cys of $\mathrm{A} 2$ globin chains are conserved in the $R$. pachyptila, Lameribrachia sp. and O. mashikoi hemoglobins. Furthermore, these Cys residues do not participate in intra- and inner-disulfide bridges. Therefore, both of the ${ }^{78}$ Cys and ${ }^{88}$ Cys residues may be the putative sulphide binding sites.

An intracellular monomeric hemoglobin I $(\mathrm{Hbl})$ of Mollusca Lucina pectinata with the molecular mass of 14,812 Da has two physiological ligands of oxygen and hydrogen sulfide, and transfers the hydrogen sulfide $\left(\mathrm{H}_{2} \mathrm{~S}\right)$ to symbiotic bacteria. In 1994, Rizzi et al. determined the crystal structure of the sulfide-reactive $L$. pectinata hemoglobin and reported highly sulfide binding affinities through the hydrogen bonding to the distal GIn and the aromatic-electrostatic interactions with Phe surrounding the heme pocket (Rizzi et al., 1994). Interestingly, B1 (d) globin chain of O. mashikoi also has a similar motif of Gln as distal His site at position 77, Phe at position 40 , and Phe at position 55 except that Phe was substituted to Val at position 81 . Therefore, B1 (d) globin chain of $O$. mashikoi may bind the hydrogen sulfide as same as L. pectinata hemoglobin.

In the present study, we first constructed the phylogentic tree including B1 (d) globin chain of $O$. mashikoi hemoglobin and a new amino acid substitution model of WAG matrix, valid for soluble proteins (Whelan and Goldman, 2001). As shown in Fig. 7, the clade of O. mashikoi B1 globin chain was branched at first from the strains $B$ with relatively high bootstrap values, suggesting that B1 globin of

Fig. 6. The multiple alignment of amino acid sequences of the globin chains from Pogonophora, Vestimentifera, Annelida, Mollusca and Vertebrata hemoglobin. The conserved amino acid residues of globin chains are indicated by asterisks (*). The specific cysteine residues used for intra-disulfide bridges (positions 7 and 152), inter-disulfide bridges (positions 6 or 143), and inferred sulfide-binding sites (positions 78,88 , and 98 ) are shaded in gray. The proximal and distal histidine residues are bold. The accession numbers of globin chains from Pogonophora, Vestimentifera, Annelida, Mollusca and Vertebrata as follows; Pogonophora Oligobrachia mashikoi: A1 (AB185392), A2 (AB185391), B1 (AB185394), B2 (AB185393). Vestimentifera Riftia pachyptila: A1 (CAD29154), A2 (P80592), B1a (CAD29156), B1b (CAD23157), B1c (CAD29158), B2 (CAD29159). Vestimentifera Lamellibrachia sp: the amino acid sequences of each globin chains (A1, A2, B1, B2) were obtained from the reference (Suzuki et al., 1995). Annelida Lumbricus terrestris: A1 (B28151), A2 (A29134), B1 (C28151), B2 (A28151). Annelida Tylorrhynchus heterochaetus: A1 (P02219), A2 (P09966), B1 (P02220), B2 (P13578). Annelida Saballa spallanzanii: B2-1 (CAC37410), B2-2 (CAC37411), A2 (CAC37412). Annelida Macrobdella decora: A1 (BAC82447), A2 (BAC82445), B1-1 (BAC82446), B1-2 (BAC82448). Annelida Tubifex tubifex: A1 (P18202). Annelida Pheretima sieboldi: A1 (P11740). Annelida Glycera dibranchiata: P1 (CAA37995), P2 (AAA29160), P3 (AAA29161), GMH4 (P15447). Mollusca Lucina pectinata: Hb1 (P41260), Hb2 (P41261), Hb3 (P41262). Human: $\alpha$-globin (AAH08572), $\beta$-globin (AAH07075), myoglobin (P02144). 


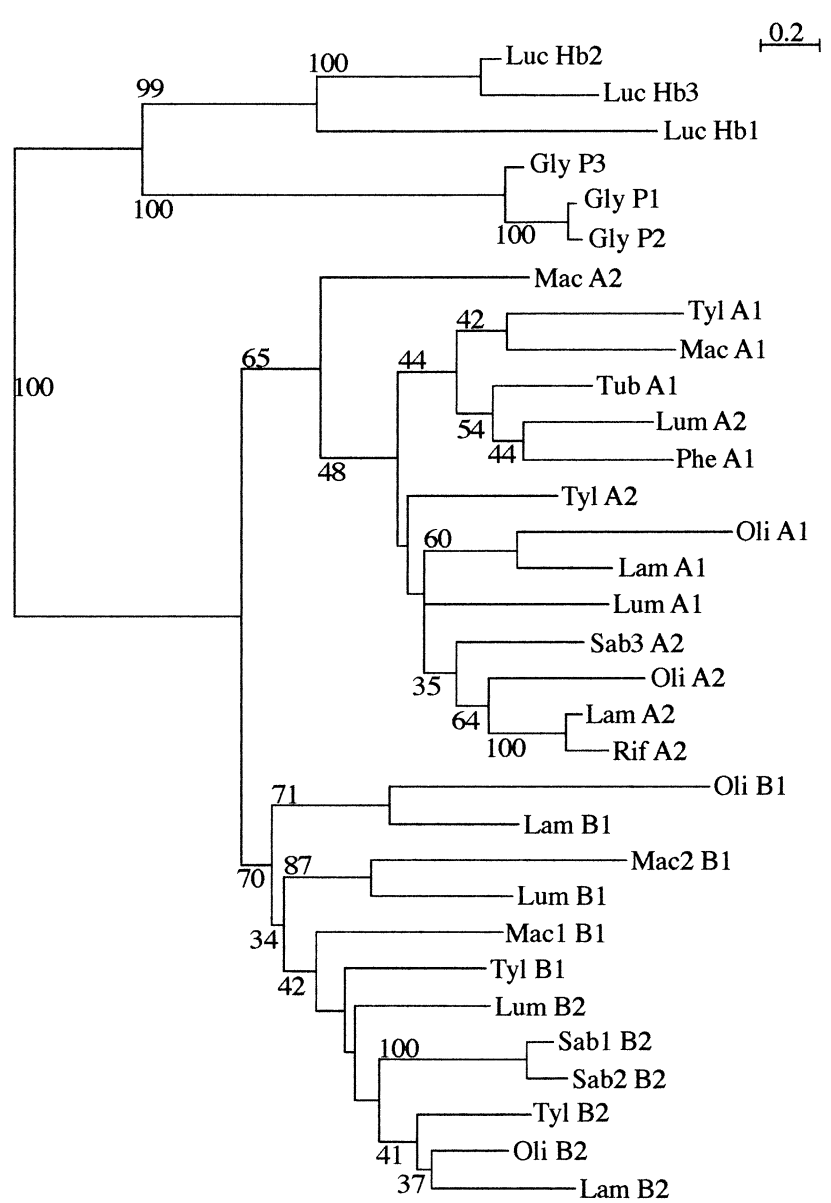

Fig. 7. The maximum likelihood consensus tree of globin chains composed of giant hemoglobin from Pogonophora Oligobrachia, Vestimentifera Lamellibrachia, Annelida Tylorrhynchus, Lumbricus, Sabella, Tubifex, Pheretima, and Macrobdella. The intracellular polymeric globin chains (P1 P3) of Annelida Glycera dibranchiata, intracellular monomeric globin chains $(\mathrm{Hb} 1)$ and homotetramer globin chains $(\mathrm{Hb} 2$ and $\mathrm{Hb} 3)$ of Mollusca Lucina pectinata were used as outgroup for rooting. The scores of branching point shows the bootstrap values derived from the 100 replicants and the scale bar shows the rate of substitution per site.

O. mashikoi might have the oldest properties in the strains B. Furthermore, B1 globin chains of ca. 350 450 kDa hemoglobins of Pogonophora $O$. mashikoi and Vestimentifera Lamellibrachia sp. were clearly separated into the cluster different from the another B1 globin cluster of ca. 3000 3600 kDa hemoglobins of Annelida Lumbricus terrestris and Macrobdella decora. The comparison between the crystal structures of $O$. mashikoi hemoglobin and Lumbricus erythrocruorin will provide quite important clues for elucidating the evolution of the extracellular giant hemoglobins.

\section{ACKNOWLEDGMENTS}

We wish to thank Masahiro Matada and the members of Noto marine laboratory, for helping with collection of $O$. mashikoi and thank Prof. Kenichiro Ishida for advice and helping with construction of phylogenetic tree. This work was supported by a Grant-in-Aid for
Scientific Research on Priority Areas (16087205) to Y. F. from the Ministry of Education, Culture, Sports, Science and Technology of Japan.

\section{REFERENCES}

Arp AJ, Childress JJ (1983) Sulfide binding by the blood of the hydrothermal vent tube worm Riftia Pachyptila. Science 219: 295-297

Arp AJ, Childress JJ, Vetter RD (1987) The sulphide-binding protein in the blood of the Vestimentiferan Tube-Worm, Riftia pachyptila, is the extracellular hemoglobin. J Exp Biol 128: 139-158

Arp AJ, Doyle ML, Cera ED, Gill SJ (1990) Oxygenation properties of the two co-occurring hemoglobins of the tube worm Riftia pachyptila. Resp physiol 80: 323-334

Bailly X, Jollivet D, Vanin S, Deutsch J, Zal F, Lallier F, Toulmond A (2002) Evolution of the sulfide-binding function within the globin multigenic family of the deep-sea hydrothermal vent tubeworm Riftia pachyptila. Mol Biol Evol 19: 1421-1433

Cavanaugh CM, Gardiner SL, Jones ML, Jannasch HW, Waterbury JB (1981) Prokaryotic cells in the hydrothermal vent tube worm Riftia Pachyptila Jones: Possible chemoautotrophic symbionts. Science 213: 340-342

Falk JE (1964) Porphyrins and metalloporphyrins. Elsevier, Amsterdam

Felbeck H (1981) Chemoautotrophic potential of the hydrothermal vent tube worm, Riftia Pachyptila Jones (Vestimentifera). Science 213: 336-338

Fountoulakis M, Juranville JF, Röder D, Evers S, Berndt P, Langen $\mathrm{H}$ (1998) Reference map of the low molecular mass protein of Haemophilus influenzae. Electrophoresis 19: 1819-1827

de Hass F, Zal F, Lallier FH, Toulmond A, Lamy JN (1996) Threedimensional reconstruction of the hexagonal bilayer hemoglobin of the hydrothermal vent tube worm Riftia pachyptila by cryoelectron microscopy. Proteins 26: 241-256

Imajima M (1973) A new species of the Genus Oligobrachia (Pogonophora) collected from Tsukumo Bay, Noto Peninsula. Ann Rep Noto Mar Lab 13: 7-12 (In Japanese with English summary)

Ivanov AV (1963) Pogonophora. Academic Press, London, 479 pp

Kawano K, Iwasaki N, Suzuki T (2003) Notable diversity in hemoglobin expression patterns among species of the deep-sea clam, Calyptogena. Cell Mol Life Sci 60: 1952-1956

Kimura H, Sato M, Sasayama Y, Naganuma, T (2003) Molecular characterization and in situ localization of endosymbiontic 16S ribosomal RNA and RuBisCO genes in the pogonophoran tissue. Mar Biotechnol 5: 261-269

Lacan P, Francina A, Souillet G, Aubry M, Couprie N, Dementhon L, Becchi M (1999) Two new alpha chain variants: Hb Boghe [alpha58 (E7)His-->Gln, alpha2], a variant on the distal histidine, and $\mathrm{Hb}$ CHarolles [alpha103 (G10) His-Tyr, alpha1]. Hemoglobin 23: 345-352

Lowry OH, Rosebrough NJ, Farr AL, Randall RJ (1951) Protein measurement with the folin phenol reagent. J Biol Chem 193: 265

Matsukawa S, Nishibu M, Nagai M, Mawatari K, Yoneyama Y (1979) Analysis of optical properties of hemoglobins in terms of the two-state model, especially from studies on abnormal hemoglobins with amino acid substitution in the $\alpha_{2} \beta_{2}$ contact region. J Biol Chem 254: 2358-2363

Matsuno A, Sasayama Y (2002) A comparative study of body wall structures of a Pogonophore and Annelid from a phylogenetic viewpoint. Zool Sci 19: 695-701

Nagai M, Sugita Y, Yoneyama Y (1972) Oxygen equilibrium and circular dichroism of hemoglobin-Rainier $\left(\alpha_{2} \beta_{2}{ }^{145 \mathrm{Tyr} \rightarrow \mathrm{Cys}}\right)$. J Biol Chem 247: 285-290 
Nelson DC, Fisher CR (1995) Chemoautotrophic and methanotrophic endosymbiotic bacteria at deep-sea vents and seeps. In "Microbiology of Deep-sea Hydrothermal vents" Ed by DM Karl, CRC Press inc. Boca Raton, pp 125-167

Rizzi M, Wittenberg JB, Coda A, Fasano M, Ascenzi P, Bolognesi M (1994) Structure of the sulfide-reactive hemoglobin from the Clam Lucina pectinata. Crystallographic analysis at $1.5 \AA$ resolution. J Mol Biol 244: 86-99

Royer Jr WE, Strand K, van Heel M, Hendrickson WA (2000) Structural hierarchy in erythrocruorin, the giant respiratory assemblage of annelids. PNAS 97: 7107-7111

Sambrook J, Fritsch EF, Maniatis T (1989) Molecular Cloning A laboratory manual, Second edition. Cold Spring Harbor Laboratory Press, Cold Spring Harbor

Sasayama Y, Matada M, Fukumori Y, Umebayashi M, Matsuno A, Nakagawa T, Imajima M (2003) External morphology of the posterior end, the "Opisthosoma" of the beard worm Oligobrachia mashikoi (Pogonophora). Zool Sci 20: 1411-1416

Schägger H, von Jagow G (1987) Tricine-sodium dodecyl sulfatepolyacrylamide gel electrophoresis for the separation of proteins in the range from 1 to $100 \mathrm{kDa}$. Anal Biochem 166: 368379

Southward EC (1982) Bacterial symbionts in Pogonophora. J Mar Biol Ass UK 62: 889-906

Strand K, Knapp JE, Bhyravbhatla B, Royer Jr WE (2004) Crystal structure of hemoglobin dodecamer from Lumbricus erythrocruorin: Allosteric core of giant Annelid respiratory complexes. $\mathrm{J}$ Mol Biol 344: 119-134

Suzuki T, Takagi T, Ohta S (1988) N-Terminal amino acid sequence of the deep-sea tube worm hemoglobin remarkably resembles that of annelid haemoglobin. Biochem J 255: 541-545

Suzuki T, Hirao Y, Vinogradov SN (1995) Primary structure of a constituent polypeptide chain of the chlorocruorin from Sabellastarte indica. Biochim Biophys Acta 1252: 189-193

Suzuki T, Vinogradov SN (2003) Globin and linker sequences of the giant extracellular hemoglobin from the Leech Macrobdella decora. J Protein Chem 22: 231-242

Svedberg T, Hedenius A (1934) The sedimentation constants of the respiratory proteins. Biol Bull And 66: 191-223

Terwilliger RC, Terwilliger NB, Hughes GM, Southward AJ, Southward EC (1987) Studies on the haemoglobins of the small Pogonophora. J Mar Biol Ass UK 67: 219-234
Vinogradov SN (1985) The structure of invertebrate extracellular hemoglobins (erythrocruorins and chlorocruorins). Comp Biochem Physiol 82B: 1-15

Vinogradov SN, Lugo SD, Mainwaring MG, Kapp OH, Crewe AV (1986) Bracelet protein: A quaternary structure proposed for the giant extarcellular hemoglobin of Lumbricus terrestris. PNAS 83: 8034-8038

Whelan S, Goldman N (2001). A general empirical model of protein evolution derived from multiple protein families using a maximum-likelihood approach. Mol Biol Evol 18: 691-699

Weber RE, Vinogradov SN (2001) Nonvertebrate hemoglobins: Functions and molecular adaptations. Physiol Rev 81: 569-628

Wells RMG, Dales RP (1976) A preliminary investigation into the oxygen-combining properties of Pogonophora haemoglobin. Comp Biochem Physiol 54A: 395-396

Yuasa HJ, Green BN, Takagi T, Suzuki N, Vinogradov SN, Suzuki T (1996) Electrospray ionization mass spectrometric composition of the $400 \mathrm{kDa}$ hemoglobin from the pogonophoran Oligobrachia mashikoi and the primary structures of three major globin chains. Biochim Biophys Acta 1296: 235-244

Zal F, Lallier FH, Wall JS, Vinogradov SN, Toulmond A (1996a) The multi-hemoglobin system of the hydrothermal vent tube worm Riftia pachyptila. I. Reexamination of the number and masses of its constituents. J Biol Chem 271: 8869-8874

Zal F, Lallier FH, Green BN, Vinogradov SN, Toulmond A (1996b) The multi-hemoglobin system of the hydrothermal vent tube worm Riftia pachyptila. II. Complete polypeptide chain composition investigated by maximum entropy analysis of mass spectra. J Biol Chem 271: 8875-8881

Zal F, Suzuki T, Kawasaki Y, Childress JJ, Lallier FH, Toulmond A (1997) Primary structure of the common polypeptide chain b from the multi-hemoglobin system of the hydrothermal vent tube worm Riftia pachyptila: An insight on the sulfide bindingsite. Proteins 29: $562-574$

Zal F, Leize E, Lallier FH, Toulmond Andre, van Dorsselaer A, Childress JJ (1998) S-Sulfohemoglobin and disulfide exchange: The mechanisms of sulfide binding by Riftia pachyptila hemoglobins. PNAS 95: 8997-9002

(Received December 20, 2004 / Accepted January 14, 2005) 